\title{
A CHARACTERIZATION OF THE RANGE OF A BOUNDED LINEAR TRANSFORMATION IN HILBERT SPACE
}

\author{
GEORGE O. GOLIGHTLY
}

\begin{abstract}
It is a theorem of Smul'jan and Mac Nerney that for $B$ a bounded linear transformation from a complete complex inner product space $\{S,(\cdot, \cdot)\}$ to $S$, with adjoint transformation $B^{*}, B(S)$ is the set of all $z$ in $S$ for which there is a nonnegative number $b$ such that for all $x$ in $S,|(z, x)|^{2}<b\left\|B^{*} x\right\|^{2}$, in which case if $w$ is that point of $(\operatorname{ker} B)^{\perp}$ such that $B w=z$ then the least such $b$ is $\|w\|^{2}$. This paper provides another description of $B(S)$ and formula for $\|w\|^{2}$.
\end{abstract}

Throughout this report, it will be supposed that $\{S,(\cdot, \cdot)\}$ is a complete complex inner product space with norm $\|\cdot\|$ and that $B$ is a bounded linear transformation from $S$ to $S$ with operator norm $\|B\| \neq 0$. The notation of the paper is consistent with that of [2].

TheORem 1. Suppose $0<B<1$. Then $B(S)$ is the set of all points $y$ in $S$ for which the series $\sum_{p=0}^{\infty}(1-B)^{p} y$ converges with respect to $\|\cdot\|$, in which case the series has limit that point $x_{1}$ of $(\operatorname{ker} B)^{\perp}$ such that $B x_{1}=y$.

Proof. Suppose $P$ is the orthogonal projection of $S$ onto $(\operatorname{ker} B)^{\perp}$ and $E_{\lambda}$ is the spectral resolution of $B$, with $E_{0_{-}}=0, E_{1}$ the identity on $S$, and $E_{\lambda}$ right-continuous at each $\lambda$. Supposing $n>0$ and $x$ in $S$, one has

$$
x-\sum_{p=0}^{n}(1-B)^{p} B x=(1-B)^{n+1} x .
$$

Thus, with $x_{1}=P x$,

$$
\left\|x_{1}-\sum_{p=0}^{n}(1-B)^{p} B x_{1}\right\|^{2}=\left\|(1-B)^{n+1} x_{1}\right\|^{2}=\int_{0-}^{1}(1-\lambda)^{2(n+1)} d\left(E_{\lambda} x_{1}, x_{1}\right) .
$$

Since $P$ commutes with $B$ and, hence, with $E_{0}, E_{0} x_{1}$ is in $\operatorname{ker}(B) \cap \operatorname{ker}(B)^{\perp}=$ $\{0\}$. Hence, the measure of $\{0\}$ with respect to the measure $\left(E(\cdot) x_{1}, x_{1}\right)$ is 0 . In view of this and the uniform boundedness and convergence to 0 on $(0,1]$ of $(1-\lambda)^{2(n+1)}$, the integral $\int_{0-}^{1}(1-\lambda)^{2(n+1)} d\left(E_{\lambda} x_{1}, x_{1}\right) \rightarrow 0$ as $n \rightarrow \infty$. Hence, $\sum_{p=0}^{\infty}(1-B)^{p} B x$ has limit $x_{1}$, the unique pre-image of $B x$ in $\operatorname{ker}(B)^{\perp}$; in other words, the vector of least norm mapped by $B$ onto $B x$.

Conversely, if $\sum_{p=0}^{\infty}(1-B)^{p} y=z$ then $(1-B) z=z-y$. Thus $y=B z$. One may note that Theorem 1 is a special case of the following. In case $E$ is a

Received by the editors May 18, 1979 and, in revised form, July 9, 1979 and August 17, 1979.

AMS (MOS) subject classifications (1970). Primary 47A30.

Key words and phrases. Complete inner product space, bounded linear transformation, nonnegative operator, spectral resolution.

(C) 1980 American Mathematical Society $0002-9939 / 80 / 0000-0369 / \$ 01.50$ 
topological vector space and $B$ a continuous linear transformation on $E$ such that for $x$ in $E B^{n} x \rightarrow 0$, then $y$ is in $(1-B)(E)$ if only if $\Sigma_{0}^{\infty} B^{p} y$ is convergent.

THEOREM 2. The range $B(S)$ of $B$ is the set of all $y$ in $S$ for which the series

$$
\|B\|^{-2} \sum_{p=0}^{\infty}\left\|\left(1-B B^{*} /\|B\|^{2}\right)^{p / 2} y\right\|^{2}
$$

converges, in which case if $x_{1}$ is that point of $(\operatorname{ker} B)^{\perp}$ such that $B x_{1}=y$ the series has limit $x_{1}$.

Proof. Suppose, initially, that $0<B<1$. Then, with the notation of the argument of Theorem 1, one has, upon replacing $B$ by $B^{2}$ in (*) and computing the inner product of both sides with $x_{1}$,

$$
\begin{aligned}
\left\|x_{1}\right\|^{2}-\sum_{p=0}^{n}\left\|\left(1-B^{2}\right)^{p / 2} B x_{1}\right\|^{2} & =\left(\left(1-B^{2}\right)^{n+1} x_{1}, x_{1}\right) \\
& =\int_{0-}^{1}\left(1-\lambda^{2}\right)^{n+1} d\left(E_{\lambda} x_{1}, x_{1}\right)
\end{aligned}
$$

the integral tending to 0 by an argument similar to that in Theorem 1.

Conversely, suppose that $\sum_{p=0}^{\infty}\left\|\left(1-B^{2}\right)^{p / 2} y\right\|^{2}<\infty$. Since, with $P y=y_{1}$ and $(1-P) y=y_{2}$,

$$
\sum_{p=0}^{n}\left\|(1-B)^{p / 2} y\right\|^{2}=\sum_{p=0}^{n}\left\{\left(\left(1-B^{2}\right)^{p} y_{1}, y_{1}\right)+\left(\left(1-B^{2}\right)^{p} y_{2}, y_{2}\right)\right\}>\sum_{p=0}^{n}\left\|y_{2}\right\|^{2},
$$

$y$ is in $\operatorname{ker}(B)^{\perp}$.

Since $\left(\sum_{p=n}^{m}\left(1-\lambda^{2}\right)^{p} \lambda\right)^{2}<\sum_{p=n}^{m}\left(1-\lambda^{2}\right)^{p}$ on $[0,1]$, the spectral theorem gives

$$
\left\|\sum_{p=n}^{m}\left(1-B^{2}\right)^{p} B y\right\|^{2}<\sum_{p=n}^{m}\left(\left(1-B^{2}\right)^{p} y, y\right)=\sum_{p=n}^{m}\left\|\left(1-B^{2}\right)^{p / 2} y\right\|^{2} \text {. }
$$

Therefore, $\sum_{p=0}^{\infty}\left(1-B^{2}\right)^{p} B y=z$ exists in $S$. Then $\left(1-B^{2}\right) z=z-B y$ so that $y-B z$ is in $\operatorname{ker}(B) \cap \operatorname{ker}(B)^{\perp}$ and $y=B z$.

In the more general case, one has the polar decomposition $B=\left(B B^{*}\right)^{1 / 2} U$ where $U$ is a partial isometry with initial space $\operatorname{ker}(B)^{\perp}$. If $z$ is in $B(S)$, then $z=B w$ for a unique $w$ in $\operatorname{ker}(B)^{\perp}$. Hence, $z=\left(B B^{*}\right)^{1 / 2}$ with $r=U w$ and $\|r\|=\|w\|$. The theorem follows from an application of the argument for the case $0<B<1$ to $\left(B B^{*}\right)^{1 / 2} /\|B\|$. Indeed, the observation that for $B$ an operator between Hilbert spaces the range of $B$ is the range of $\left(B B^{*}\right)^{1 / 2}$ provides a two-space analogue of the theorem.

The author is grateful to the referee for several helpful suggestions.

\section{REFERENCES}

1. J. S. Mac Nerney, Hermitian moment sequences, Trans. Amer. Math. Soc. 103 (1962), 45-81.

2. F. Riesz and B. Sz.-Nagy, Functional analysis, Ungar, New York, 1955.

3. Ju. L. Smul'jan, Two-sided division in a ring of operators, Mat. Zametki 1 (1967), 605-610.

Department of Mathimatics, Auburn University, Auburn, Alabama 36830 